\title{
TWO-DIMENSIONAL OPTIMIZATION PROBLEM OF PLANT LOCATION
}

\author{
Tran Gia Lich(1), Phan NGOC VinH(2) \\ (1) Institute of Mathematics, P.O Box 631, BoHo, $10000 \mathrm{Ha} \mathrm{Noi}$ \\ (2) Institute of Mechanics, $264 \mathrm{Doi}$ Can, $\mathrm{Ha} \mathrm{Noi}$
}

\begin{abstract}
In this paper, the following matters are presented: the adjoint problem of the two-dimensional matter propagation problem; the algorithm for determination of a domain in which a plant can be located so that the values of the pollution-level reflecting functional does not exceed a given value at considered sensitive areas; application of this algorithm for numerical experiments to a typical problem.
\end{abstract}

1. Equation of the suspended matter propagation and its adjoint equation (see [1])

The equation of the suspended matter propagation, i.e. the matter transport and diffusion equation in the horizontal 2D case has the following form:

$$
\frac{\partial C}{\partial t}+u \frac{\partial C}{\partial x}+v \frac{\partial C}{\partial y}+\sigma C=f+\gamma \Delta C, \quad(x, y) \in G, 0 \leq t \leq T
$$

with the initial and boundary conditions:

$$
\left.C\right|_{t=0}=C^{0},\left.\quad C\right|_{\Gamma^{-}}=\varphi,\left.\quad \frac{\partial C}{\partial n}\right|_{\Gamma^{+}}=0
$$

where $x, y, t$ are the space and time variables; $C$ is the matter concentration; $\sigma$ is the decay coefficient; $f$ is the source intensity; $\gamma$ is the diffusion coefficient; $u, v$ are respectively velocity components in the $x$ and $y$ directions, and satisfy the following equation:

$$
\frac{\partial u}{\partial x}+\frac{\partial v}{\partial y}=0
$$

$\Gamma=\Gamma^{+}+\Gamma^{-}$with $\Gamma^{+}$is the boundary part, at which $u_{n} \geq 0 ; \Gamma^{-}$is the boundary part, at which $u_{n}<0 ; \Delta=\frac{\partial^{2}}{\partial x^{2}}+\frac{\partial^{2}}{\partial y^{2}}$-Laplace operator; $u_{n}$ is the projection of the velocity on the external normal vector $\vec{n}$.

Using (1.3), the equation (1.1) can be rewritten as follows:

$$
\frac{\partial C}{\partial t}+\frac{\partial u C}{\partial x}+\frac{\partial v C}{\partial y}+\sigma C=f+\gamma \Delta C
$$


Solution of the equation (1.1) may be determined under the form: $C=C_{1}+C_{2}$ where, $C_{1}$ and $C_{2}$ are the solutions of the following equation:

$$
\frac{\partial C_{1}}{\partial t}+u \frac{\partial C_{1}}{\partial x}+v \frac{\partial C_{1}}{\partial y}+\sigma C_{1}=\gamma \Delta C_{1}
$$

with the initial and boundary conditions:

$$
\left.C_{1}\right|_{t=0}=C^{0},\left.\quad C_{1}\right|_{\Gamma^{-}}=\varphi,\left.\quad \frac{\partial C_{1}}{\partial n}\right|_{\Gamma^{+}}=0
$$

and

$$
\frac{\partial C_{2}}{\partial t}+\frac{\partial u C_{2}}{\partial x}+\frac{\partial v C_{2}}{\partial y}+\sigma C_{2}=\gamma \Delta C_{2}+f
$$

with the initial and boundary conditions:

$$
\left.C_{2}\right|_{t=0}=0,\left.\quad C_{2}\right|_{\Gamma^{-}}=0,\left.\quad \frac{\partial C_{2}}{\partial n}\right|_{\Gamma^{+}}=0 .
$$

We now establish the adjoint equation of the equation (1.6). By multiplying both sides of the equation (1.6) by a some function $C_{2}^{*}$ and integrating the equation obtained on the area $G \times[0, T]$, we get:

$$
\begin{aligned}
\int_{0}^{T} d t \int_{G} C_{2}^{*} \frac{\partial C_{2}}{\partial t} d G+\int_{0}^{T} d t \int_{G} C_{2}^{*} \operatorname{div}\left(\vec{u} C_{2}\right) d G \\
\quad+\int_{0}^{T} d t \int_{G} \sigma C_{2} C_{2}^{*} d G-\gamma \int_{0}^{T} d t \int_{G} C_{2}^{*} \Delta C_{2} d G=\int_{0}^{T} d t \int_{G} C_{2}^{*} f d G .
\end{aligned}
$$

Let $\gamma=$ const, using the partial integration technique, the Green formula and the condition (1.3), we have:

$$
\begin{aligned}
& \int_{0}^{T} d t \int_{G} C_{2}^{*} \frac{\partial C_{2}}{\partial t} d G=\left.\int_{G} C_{2}^{*} C_{2}\right|_{0} ^{T} d G-\int_{0}^{T} d t \int_{G} C_{2} \frac{\partial C_{2}^{*}}{\partial t} d G, \\
& \int_{0}^{t} d t \int_{G} C_{2}^{*} \operatorname{div}\left(\vec{u} C_{2}\right) d G=\int_{0}^{T} d t \int_{\Gamma} u_{n} C_{2}^{*} C_{2} d \Gamma-\int_{0}^{T} d t \int_{G} C_{2} \operatorname{div}\left(\vec{u} C_{2}^{*}\right) d G, \\
& \gamma \int_{0}^{T} d t \int_{G} C_{2}^{*} \Delta C_{2} d G=\gamma \int_{0}^{T} d t \int_{\Gamma}\left(C_{2}^{*} \frac{\partial C_{2}}{\partial n}-C_{2} \frac{\partial C_{2}^{*}}{\partial n}\right) d \Gamma+\gamma \int_{0}^{T} d t \int_{G} C_{2} \Delta C_{2}^{*} d G .
\end{aligned}
$$


Putting these expressions into (1.8), one deduces:

$$
\begin{aligned}
& \int_{0}^{T} d t \int_{G} C_{2}\left(-\frac{\partial C_{2}^{*}}{\partial t}-\operatorname{div}\left(\vec{u} C_{2}^{*}\right)+\sigma C_{2}^{*}-\gamma \Delta C_{2}^{*}\right) d G= \\
& =\int_{0}^{T} d t \int_{G} C_{2}^{*} f d G-\left.\int_{G} C_{2}^{*} C_{2}\right|_{t=T} d G+\left.\int_{G} C_{2}^{*} C_{2}\right|_{t=0} d G-\int_{0}^{T} d t \int_{\Gamma} u_{n} C_{2} C_{2}^{*} d \Gamma \\
& \quad+\gamma \int_{0}^{T} d t \int_{\Gamma}\left(C_{2}^{*} \frac{\partial C_{2}}{\partial n}-C_{2} \frac{\partial C_{2}^{*}}{\partial n}\right) d \Gamma .
\end{aligned}
$$

Let the function $C_{2}^{*}$ satisfy the following equation:

$$
-\frac{\partial C_{2}^{*}}{\partial t}-\operatorname{div}\left(\vec{u} C_{2}^{*}\right)+\sigma C_{2}^{*}-\gamma \Delta C_{2}^{*}=p .
$$

From the initial and the boundary conditions (1.7), one yields:

$$
\begin{aligned}
& \left.\int_{G} C_{2} C_{2}^{*}\right|_{t=0} d G=0, \\
& \int_{0}^{T} d t \int_{\Gamma} u_{n} C_{2} C_{2}^{*} d \Gamma=\int_{0}^{T} d t \int_{\Gamma^{+}} u_{n} C_{2} C_{2}^{*} d \Gamma, \\
& \gamma \int_{0}^{T} d t \int_{\Gamma}\left(C_{2}^{*} \frac{\partial C_{2}}{\partial n}-C_{2} \frac{\partial C_{2}^{*}}{\partial n}\right) d \Gamma=\gamma \int_{0}^{T} d t \int_{\Gamma^{+}}\left(-C_{2} \frac{\partial C_{2}^{*}}{\partial n}\right) d \Gamma+\gamma \int_{0}^{T} d t \int_{\Gamma^{-}} C_{2}^{*} \frac{\partial C_{2}}{\partial n} d \Gamma .
\end{aligned}
$$

From the above expressions and (1.10), the equation (1.9) can be rewritten under the form:

$$
\begin{aligned}
\int_{0}^{T} d t \int_{G} p C_{2} d G= & \int_{0}^{T} d t \int_{G} f C_{2}^{*} d G+\left.\int_{G} C_{2} C_{2}^{*}\right|_{t=T} d G \\
& +\gamma \int_{0}^{T} d t \int_{\Gamma^{-}} C_{2}^{*} \frac{\partial C_{2}}{\partial n} d \Gamma-\int_{0}^{T} d t \int_{\Gamma^{+}} C_{2}\left(\gamma \frac{\partial C_{2}^{*}}{\partial n}+u_{n} C_{2}^{*}\right) d \Gamma .
\end{aligned}
$$

Let the initial and boundary conditions of the equation (1.10) be chosen as follows:

$$
\left.C_{2}^{*}\right|_{t=T}=0,\left.\quad C_{2}^{*}\right|_{\Gamma^{-}}=0,\left.\quad\left(\gamma \frac{\partial C_{2}^{*}}{\partial n}+u_{n} C_{2}^{*}\right)\right|_{\Gamma^{+}}=0
$$


Then, from (1.11) and (1.12) we get the dual form:

$$
\int_{0}^{T} d t \int_{G} p C_{2} d G=\int_{0}^{T} d t \int_{G} f C_{2}^{*} d G .
$$

It is easy to verify that the problem $(1.10),(1.12)$ is the adjoint problem of the (1.6), (1.7). Indeed, with the notation:

$$
A=\frac{\partial}{\partial t}+\frac{\partial u \cdot}{\partial x}+\frac{\partial v \cdot}{\partial y}+\sigma-\gamma \Delta \quad A^{*}=-\frac{\partial}{\partial t}-\frac{\partial u}{\partial x}-\frac{\partial v}{\partial y}+\sigma-\gamma \Delta,
$$

we have:

$$
\begin{aligned}
& A C_{2}=f, \quad A^{*} C_{2}^{*}=p \\
& \left(A C_{2}, C_{2}^{*}\right)=\left(f, C_{2}^{*}\right)=\int_{0}^{T} d t \int_{G} f C_{2}^{*} d G=\int_{0}^{T} d t \int_{G} p C_{2} d G=\left(C_{2}, p\right)=\left(C_{2}, A^{*} C_{2}^{*}\right) .
\end{aligned}
$$

Use of the variable transformation $t_{1}=T-t$, the equation (1.10) becomes:

$$
\begin{aligned}
& \frac{\partial C_{2}^{*}}{\partial t_{1}}-\operatorname{div}\left(\vec{u} C_{2}^{*}\right)+\sigma C_{2}^{*}-\gamma \Delta C_{2}^{*}=p, \\
& \left.C_{2}^{*}\right|_{t_{1}=0}=0,\left.\quad C_{2}^{*}\right|_{\Gamma^{-}}=0,\left.\quad\left(\gamma \frac{\partial C_{2}^{*}}{\partial n}+u_{n} C_{2}^{*}\right)\right|_{\Gamma^{+}}=0 .
\end{aligned}
$$

For simplicity, by using (1.3), we obtain an another form of the adjoint equation (1.14):

$$
\frac{\partial C_{2}^{*}}{\partial t_{1}}-u \frac{\partial C_{2}^{*}}{\partial x}-v \frac{\partial C_{2}^{*}}{\partial y}+\sigma C_{2}^{*}-\gamma \Delta C_{2}^{*}=p
$$

2. Pollution-level reflecting functionals (see [1])

Assume that the suspended matter concentration $C$ is calculated from the equation (1.1). We consider the following functionals:

a. The time-averaged amount of the matter concentration $C$ on a sensitive area $G_{k} \subset G$ for the period $T: J_{k}^{A}=\frac{1}{T} \int_{0}^{T} d t \int_{G_{k}} C d G$.

b. The total amount of settling matter in the same area $G_{k} \subset G: J_{k}^{B}=\int_{0}^{T} d t \int_{G_{k}} a C d G$, where, the constant a represents portion of matter which settles down, that are mainly the heavy matters and partly the suspended matters settling down by downward diffusion. 
c. Generalized functional:

$$
J_{k}=\int_{0}^{T} d t \int_{G_{k}} p C d G \text { where } \quad p= \begin{cases}\frac{1}{T}+a, & (x, y) \in G_{k} \\ 0, & (x, y) \notin G_{k}\end{cases}
$$

and $p$ is a function referring to the economic, sanitary, ecological, health standards and so on.

d. Global functional:

$$
Y_{p}=\int_{0}^{T} d t \int_{G} p C d G \quad \text { where, } \quad p= \begin{cases}\frac{1}{T}+G_{k}, & (x, y) \in G_{k}, k=1,2, \ldots, m \\ 0, & (x, y) \notin \bigcup_{k=1}^{m} G_{k} .\end{cases}
$$

\section{Optimization problem of plant location (see [1])}

Let $G_{k}(k=1,2, \ldots, m)$ be considered areas, recreation zones or other environmentally sensitive areas on the region $G$. Our problem is to determine the domain $\Omega_{k} \subset G$ so that the pollution matter from a plant located in this domain $\Omega_{k}$ satisfies the following condition for the sensitive area $G_{k}$ :

$$
Y_{k}=\int_{0}^{T} d t \int_{G_{k}} p C d G \leq \bar{c}_{k}, \quad \text { where } \quad p= \begin{cases}\frac{1}{T}+a_{k}, & (x, y) \in G_{k} \\ 0, & (x, y) \notin G_{k}\end{cases}
$$

and $\bar{c}_{k}$ is a given figure.

If the determination of domain $\Omega_{k}$ is impossible on the $G$, the reduction of rate of the pollution emission $Q$, will make the determination of the plant location possible.

Assume that on the region $G$ there are $\mathrm{m}$ sensitive areas $G_{k}(k=1, \ldots, m)$ and the source of matter emission is located at a point $r_{0}=\left(x_{0}, y_{0}\right)$. Then, the source intensity can be described by the function: $f(x, y)=Q \delta\left(r-r_{0}\right), \quad Q=$ const where, $\delta(r)=\left\{\begin{array}{ll}\infty, & r=r_{0} \\ 0, & r \neq r_{0}\end{array}\right.$ is Dirac function, and from (1.1), we get:

$$
\frac{\partial C}{\partial t}+u \frac{\partial C}{\partial x}+v \frac{\partial C}{\partial y}+\sigma C=Q \delta\left(r-r_{0}\right)+\gamma \Delta C
$$

with the conditions: $\left.C\right|_{t=0}=C^{0},\left.\quad C\right|_{\Gamma^{-}}=\varphi,\left.\quad \frac{\partial C}{\partial n}\right|_{\Gamma^{+}}=0$.

In order to determine the domain $\Omega$, in which the plant can be located so that in all sensitive areas $G_{k}$, the generalized functional $Y_{k}$ satisfies the condition (3.1), we do as follows: 
a. Calculation of concentration $C$ from the equation (1.5):

$$
\frac{\partial C}{\partial t}+u \frac{\partial C}{\partial x}+v \frac{\partial C}{\partial y}+\sigma C=\gamma \Delta C
$$

with the initial and boundary conditions $\left.C\right|_{t=0}=C^{0},\left.\quad C\right|_{\Gamma^{-}}=\varphi,\left.\quad \frac{\partial C}{\partial n}\right|_{\Gamma^{+}}=0$ and generalized functional (2.1): $J_{k}=\int_{0}^{T} d t \int_{G_{k}} p C d G=\widetilde{c}_{k}$.

b. Solving $m$ adjoint equations:

$$
\frac{\partial C_{k}^{*}}{\partial t_{1}}-u \frac{\partial C_{k}^{*}}{\partial x}-v \frac{\partial C_{k}^{*}}{\partial y}+\sigma C_{k}^{*}-\gamma \Delta C_{k}^{*}=p_{k}
$$

where, $p_{k}=\left\{\begin{array}{ll}\frac{1}{T}+a_{k}, & (x, y) \in G_{k} \\ 0, & (x, y) \notin G_{k}\end{array}\right.$ with the conditions:

$$
\left.C_{k}^{*}\right|_{t_{1}=0}=0,\left.\quad C_{k}^{*}\right|_{\Gamma^{-}}=0,\left.\quad\left(v \frac{\partial C_{k}^{*}}{\partial n}+u_{n} C_{k}^{*}\right)\right|_{\Gamma^{+}}=0,
$$

we obtain the solutions $C_{k}^{*}(k=1,2, \ldots, m)$.

From the dual form (1.13), we get:

$$
\begin{aligned}
Y_{k}^{*} & =\int_{0}^{T} d t \int_{G} p_{k} C d G=\int_{0}^{T} d t \int_{G} Q \delta\left(r-r_{0}\right) C_{k}^{*} d G \\
& =\int_{0}^{T} Q C_{k}^{*}\left(r_{0}, t\right) d t=\int_{0}^{T} Q C_{k}^{*}\left(r_{0}, T-t_{1}\right) d t_{1}
\end{aligned}
$$

which must satisfy the condition: $Y_{k}^{*} \leq \bar{c}_{k}-\widetilde{c}_{k}=\overline{\bar{c}}_{k}$.

Now we consider the function: $Y_{k}^{*}(r)=Q \int_{0}^{T} C_{k}^{*}(r, t) d t$ and draw the iso-grams of $Y_{k}^{*}(r)=$ const. Then, $\Omega_{k}$ in which the functional $Y_{k}^{*}(r) \leq \overline{\bar{c}}_{k}$ are found out. If there is perchance no area $\Omega_{k}$ inside $G$, it may be re-established anyway by reducing the discharge intensity $Q$.

c. Overlaying all the areas $\Omega_{k}(k=1, \ldots, m)$, we obtain the domain $\Omega,\left(\Omega=\bigcap_{k=1}^{m} \Omega_{k}\right)$. $\Omega$ will be the domain in which the plant can be located so that pollution standards will be met in all the areas $G_{k} \subset G,(k=1,2, \ldots, m)$.

\section{Algorithm (see [2]-[4])}

The equation (1.5) and the adjoint equation (1.15) may be rewritten in a common form: 


$$
\frac{\partial C}{\partial t}+\Lambda C=f
$$

where, $\Lambda=\Lambda_{1}+\Lambda_{2}, \Lambda_{1}= \pm u \frac{\partial}{\partial x}-\gamma \frac{\partial^{2}}{\partial x^{2}}+\frac{\sigma}{2}, \Lambda_{2}= \pm v \frac{\partial}{\partial y}-\gamma \frac{\partial^{2}}{\partial y^{2}}+\frac{\sigma}{2}$.

Equation (4.1) is solved by the method of the directional decomposition (splitting method):

$$
\begin{gathered}
\frac{C^{k+1}-C^{k}}{d t}+\Lambda\left[\theta C^{k+1}+(1-\theta) C^{k}\right]=f^{k+1} \\
\text { or } \quad(I+d t \theta \Lambda) C^{k+1}=[I-d t(1-\theta) \Lambda] C^{k}+d t f^{k+1},
\end{gathered}
$$

where $0 \leq \theta \leq 1, I$ is the unique operator.

Using approximation:

$$
\left[I+d t \theta\left(\Lambda_{1}+\Lambda_{2}\right)\right]=\left(I+d t \theta \Lambda_{1}\right)\left(I+d t \theta \Lambda_{2}\right)+0\left(d t^{2}\right)
$$

from (4.2), one deduces:

$$
\left(I+d t \theta \Lambda_{1}\right)\left(I+d t \theta \Lambda_{2}\right) C^{k+1}=d t f^{k+1}+[I-d t(1-\theta) \Lambda] C^{k} .
$$

The computational process contains two steps:

$$
\begin{aligned}
\left(I+d t \theta \Lambda_{1}\right) C^{k+1 / 2} & =[I-d t(1-\theta) \Lambda] C^{k}+d t f^{k+1} \\
\left(I+d t \theta \Lambda_{2}\right) C^{k+1} & =C^{k+1 / 2}
\end{aligned}
$$

a. Discretizing the equation (4.3) by an implicit finite difference scheme:

$$
\begin{aligned}
& \Lambda_{1} C^{k+1 / 2}=\frac{( \pm u+|u|)_{m, n}^{k+1 / 2}}{2} \frac{\left(C_{m, n}^{k+1 / 2}-C_{m-1, n}^{k+1 / 2}\right)}{\Delta x} \\
& +\frac{( \pm u-|u|)_{m, n}^{k+1 / 2}}{2} \frac{\left(C_{m+1, n}^{k+1 / 2}-C_{m, n}^{k+1 / 2}\right)}{\Delta x}-\gamma \frac{\left(C_{m+1, n}^{k+1 / 2}-2 C_{m, n}^{k+1 / 2}+C_{m-1, n}^{k+1 / 2}\right)}{\Delta x^{2}}+\frac{\sigma}{2} \\
& \Lambda C^{k}= \pm u_{m, n}^{k+1 / 2} \frac{\left(C_{m+1, n}^{k}-C_{m-1, n}^{k}\right)}{2 \Delta x}-\gamma \frac{C_{m+1, n}^{k}-2 C_{m, n}^{k}+C_{m-1, n}^{k}}{\Delta x^{2}} \\
& \quad \pm v_{m, n}^{k+1 / 2} \frac{\left(C_{m, n+1}^{k}-C_{m, n-1}^{k}\right)}{2 \Delta y}-\gamma \frac{C_{m, n+1}^{k}-2 C_{m, n}^{k}+C_{m, n-1}^{k}}{\Delta y^{2}}+\sigma
\end{aligned}
$$

we obtain:

$$
a_{m} C_{m+1, n}^{k+1 / 2}+b_{m} C_{m, n}^{k+1 / 2}+c_{m} C_{m-1, n}^{k+1 / 2}=d_{m},
$$


where,

$$
\begin{aligned}
& a_{m}=\frac{( \pm u-|u|)_{m, n}^{k+1 / 2} \theta d t}{2 \Delta x}-\frac{\gamma \theta d t}{(\Delta x)^{2}}, \quad b_{m}=1+\frac{\theta|u|_{m, n}^{k+1 / 2} d t}{\Delta x}+2 \frac{\gamma \theta d t}{(\Delta x)^{2}}+\frac{\sigma d t}{2} \\
& c_{m}=-\frac{( \pm u+|u|)_{m, n}^{k+1 / 2} \theta d t}{2 \Delta x}-\frac{\gamma \theta d t}{(\Delta x)^{2}}, \quad d_{m}=d t f_{m, n}^{k+1}+[I-d t(1-\theta) \Lambda] C_{m, n}^{k} .
\end{aligned}
$$
$\delta>0$.

It is easy to verify that: $b_{m}>0, a_{m}<0, c_{m}<0$ and $\left|b_{m}\right| \geq\left|a_{m}\right|+\left|c_{m}\right|+\delta$,

So, the linear equation system (4.5) has the unique solution and the computational error of the following double sweep method

$$
C_{m, n}^{k+1}=L_{m} C_{m+1, n}^{k+1}+K_{m}
$$

where, $L_{m}=\frac{-a_{m}}{b_{m}+c_{m} L_{m-1}}, K_{m}=\frac{d_{m}-c_{m} K_{m-1}}{b_{m}+c_{m} L_{m-1}}$, is not accumulated (see [5]).

b. Discretizing the equation (4.4) by a difference scheme:

$$
\begin{aligned}
& \Lambda_{2} C^{k+1}= \frac{( \pm v+|v|)_{m, n}^{k+1}}{2} \frac{\left(C_{m, n}^{k+1}-C_{m, n-1}^{k+1}\right)}{\Delta y}+\frac{( \pm v-|v|)_{m, n}^{k+1}}{2} \frac{\left(C_{m, n+1}^{k+1}-C_{m, n}^{k+1}\right)}{\Delta y} \\
&-\gamma \frac{\left(C_{m, n+1}^{k+1}-2 C_{m, n}^{k+1}+C_{m, n-1}^{k+1}\right)}{\Delta y^{2}}+\frac{\sigma}{2} C_{m, n}^{k+1}
\end{aligned}
$$

we also get:

$$
\tilde{a}_{n} C_{m, n+1}^{k+1}+\tilde{b}_{n} C_{m, n}^{k+1}+\tilde{c}_{n} C_{m, n-1}^{k+1}=\tilde{d}_{n}
$$

where,

$$
\begin{aligned}
& \tilde{a}_{m}=\frac{( \pm v-|v|)_{m, n}^{k+1} \theta d t}{2 \Delta y}-\frac{\gamma \theta d t}{(\Delta y)^{2}}, \quad \tilde{b}_{m}=1+\frac{\theta|v|_{m, n}^{k+1} d t}{\Delta y}+2 \frac{\gamma \theta d t}{(\Delta y)^{2}}+\frac{d t \sigma}{2} \\
& \tilde{c}_{m}=-\frac{( \pm v+|v|)_{m, n}^{k+1} d t}{2 \Delta y}-\frac{\gamma \theta d t}{(\Delta y)^{2}}, \quad \tilde{d}_{m}=C_{m, n}^{k+1 / 2} .
\end{aligned}
$$

Obviously: $\tilde{b}_{m}>0, \tilde{a}_{m}<0, \tilde{c}_{m}<0$ and $\left|\tilde{b}_{m}\right| \geq\left|\tilde{a}_{m}\right|+\left|\tilde{c}_{m}\right|+\delta, \delta>0$.

Also, the equation system (4.7) has the unique solution and the double sweep method (4.6) does not produce an accumulated computational error.

\section{Numerical experiments}

The mentioned-above algorithm is applied to solve the following optimization problem of plant location: 
- The computed rectangular region $G=1000 m \times 1000 m$ is covered by a uniform grid $51 \times 51$ with spacing steps: $d x=20 \mathrm{~m}, d y=20 \mathrm{~m}$.

- A constant velocity field $(u, v): u=0.5 \mathrm{~m} / \mathrm{s}, v=-0.5 \mathrm{~m} / \mathrm{s}$.

- Diffusion coefficient : $\gamma=0.5 \mathrm{~m}^{2} / \mathrm{s}$.

- Decay coefficient: $\sigma=0.0005 \mathrm{~s}^{-1}$.

- Time step: $d t=5 \mathrm{~s}$.

- Time simulation: $T=20000 \mathrm{~s}$.

- 3 considered sensitive rectangular areas $G_{k}$ inside $G(k=1,2,3)$ with the left-bottom corner coordinates and the right-top corner coordinates are as follows:

$+G_{1}=[(24.5,8.5),(25.5,9.5)],+G_{2}=[(37.5,12.5),(39.5,14.5)]$,

$+G_{3}=[(29.5,33.5),(30.5,34.5)]$.

- Standard concentration: $\overline{\bar{c}}_{k}=10 \mathrm{mg} / \mathrm{l}(k=1,2,3)$.

The numerical results are illustrated in Fig. 1. In this figure, the figure on the

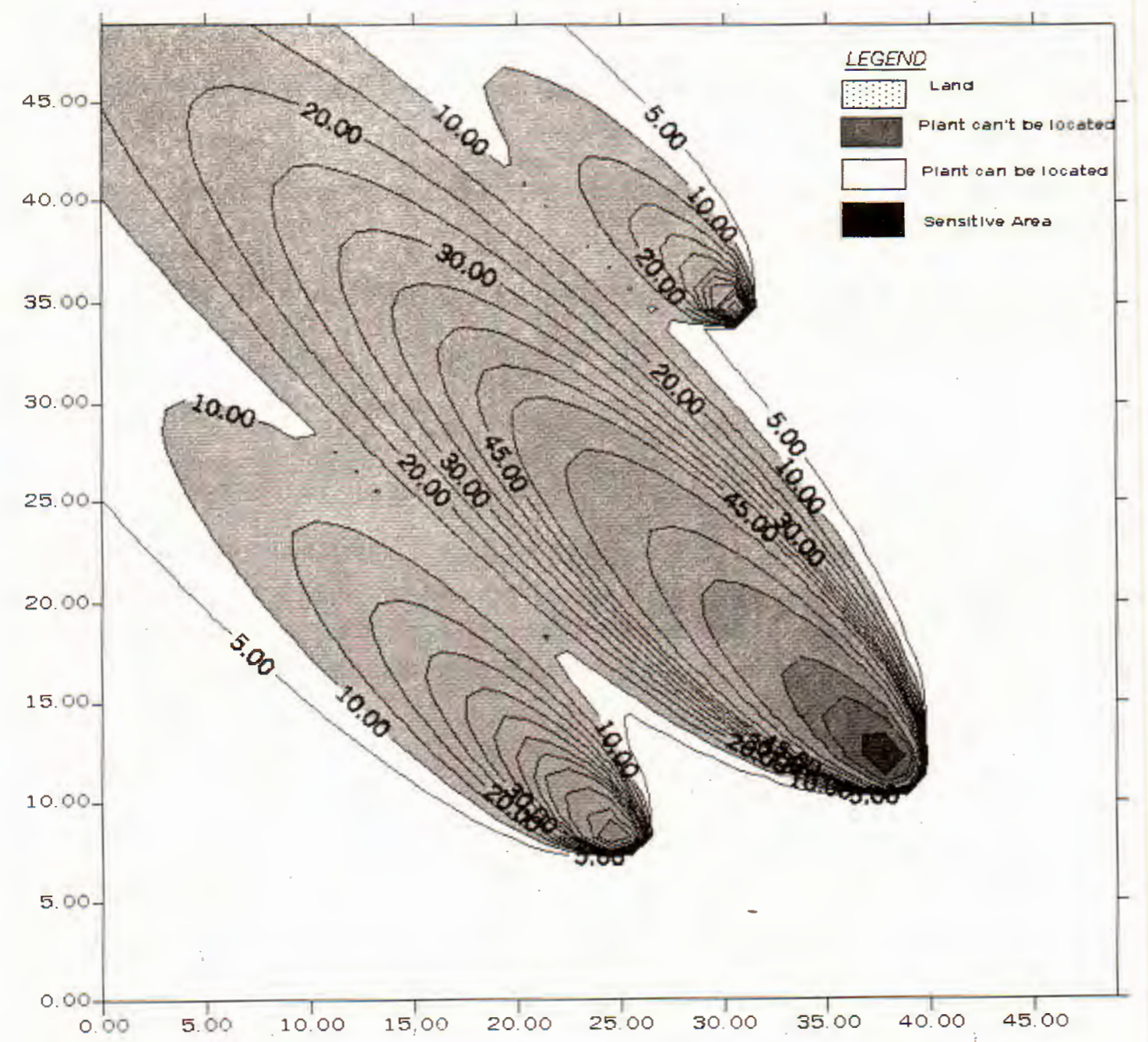

Fig. 1. Distribution of value of the pollution level-reflecting functionals $Y_{k}^{*}$ for problem 1 
contour lines indicates value of the pollution-level reflecting functionals $Y_{k}^{*}$. As a result, the domain $\Omega$ where the plant can be located so that the sanitary condition in the all areas $G_{k}$ are satisfied (that means $Y_{k}^{*} \leq \overline{\bar{c}}_{k}$ ) is in white.

Acknowledgements. This work is partly supported by the Council for Natural Sciences of Vietnam, by the Program "Applied Mathematics" NCNST and the Basic Scientific Research Program NCNST.

\section{REFERENCES}

1. Marchuk G. I. Adjoint equations and analysis of complex systems. Publishers NAUKA, Moscow, 1992 (In Russian).

2. Kovenia V: M, Yanenko N. N: Method of decomposition in the problems of the gas dynamics. Publishers NAUKA, Novosibirsk, 1981 (In Russian).

3. Marchuk G. I. Method of decomposition. Publishers NAUKA, Moscow, 1988 (In Russian).

4. Marchuck G. I. Method of Computational Mathematics. Publishers NAUKA, Moscow, 1980 (In Russian).

5. Godunov S. K, Rjabenki V. S. Difference Schemes. Publishers NAUKA, Moscow; 1988 (In Russian).

Received December 14, 2000

\section{BÀI TOÁN 2 CHIỀU TỐI UUU XÁC ĐỊNH VI TRÍ NGUỒN THẢI}

Bài báo trình bày các vấn đề sau: Bài toán liên hợp với bài toán lan truyền vật chất 2 chiều. Thuật toán xác định miền có thể đặt xí nghiệp sao cho phiến hàm biểu thị mức độ ô nhiễm không vượt quá mức độ cho phép ớ các vùng nhạy cảm quan tầm. Đã áp dụng thuật toán này dể tính toán cho một bài toán mẫu. 